Review Article

\title{
Stability and Hopf Bifurcation of a Generalized Chikungunya Virus Infection Model with Two Modes of Transmission and Delays
}

\author{
Hajar Besbassi $\left(\mathbb{D},{ }^{1}\right.$ Khalid Hattaf $\left(\mathbb{D},{ }^{1,2}\right.$ and Noura Yousfi ${ }^{1}$ \\ ${ }^{1}$ Laboratory of Analysis, Modeling and Simulation (LAMS), Faculty of Sciences Ben M'sik, Hassan II University of Casablanca, \\ P.O Box 7955 Sidi Othman, Casablanca, Morocco \\ ${ }^{2}$ Centre Régional des Métiers de l'Education et de la Formation (CRMEF), Derb Ghalef, Casablanca 20340, Morocco
}

Correspondence should be addressed to Hajar Besbassi; hajar.besbassi@gmail.com

Received 6 June 2020; Revised 9 August 2020; Accepted 28 September 2020; Published 15 October 2020

Academic Editor: Abdul Qadeer Khan

Copyright (C) 2020 Hajar Besbassi et al. This is an open access article distributed under the Creative Commons Attribution License, which permits unrestricted use, distribution, and reproduction in any medium, provided the original work is properly cited.

A generalized chikungunya virus (CHIKV) infection model with nonlinear incidence functions and two time delays is proposed and investigated. The model takes into account both modes of transmission that are virus-to-cell infection and cell-to-cell transmission. Furthermore, the local and global stabilities of the disease-free equilibrium and the chronic infection equilibrium are established by using the linearization and Lyapunov functional methods. Moreover, the existence of Hopf bifurcation is also analyzed. Finally, an application is presented in order to support the analytical results.

\section{Introduction}

The CHIKV belongs to the family Togaviridae, a term built from the Roman toga, to describe the draped appearance of their envelope [1]. Its genetic material consists of a singlestranded, thermosensitive RNA, about 15,000 nucleotides long. The multiplication of the viral genome in the cell is not strictly accurate, a common property of RNA viruses, which results in mutations that can affect not only the infective and pathogenic powers of the virus but also its passage from one kind of Aedes to another. Viral RNA of the infecting virion is included in a spherical particle made up of viral or nucleocapsid proteins assembled regularly and is of a size of around 70 nanometres. The virus multiplies with great ease in vitro, but also in vivo in mosquito cells, which explains the high infective power of contaminated Aedes. The female mosquito infects itself during a blood meal (necessary for laying) on a contaminated individual (man especially in the epidemic phase and also bats, monkeys, and other vertebrates). The virus proliferates in the insect. It is injected into a man or animal during a subsequent blood meal, during the initial phase of the bite, which includes the injection of "saliva" from the infected insect, before the blood meal itself [2].

In [3], the authors described the CHIKV replication cycle (see Figure 1) and the results of chikungunya virus infection particularly intense joint and muscle pain that forces patients to lean forward. After about one week of incubation, the pain appears, especially in the wrists, fingers, knees, ankles, and feet. The hips and shoulders are more rarely affected. These pains are accompanied by severe headaches, fever (over $38.5^{\circ} \mathrm{C}$ ), and rash in the chest and limbs, as well as lymph node swelling and conjunctivitis. Other symptoms sometimes appear, including bleeding of the gums or nose and neurological disorders.

Medical management is purely symptomatic, based on pain and anti-inflammatory treatments. However, these treatments have no preventive effect on the occurrence of a chronic evolution. First isolated in Uganda in 1953, CHIKV circulates mainly in the intertropical zone. This disease is particularly virulent in Africa and South Asia. However, cases were detected in the French territory as early as 2010 (in the south of France) and in 2013 and 2014 in the West Indies [4]. It can be responsible for important epidemics [5]. 


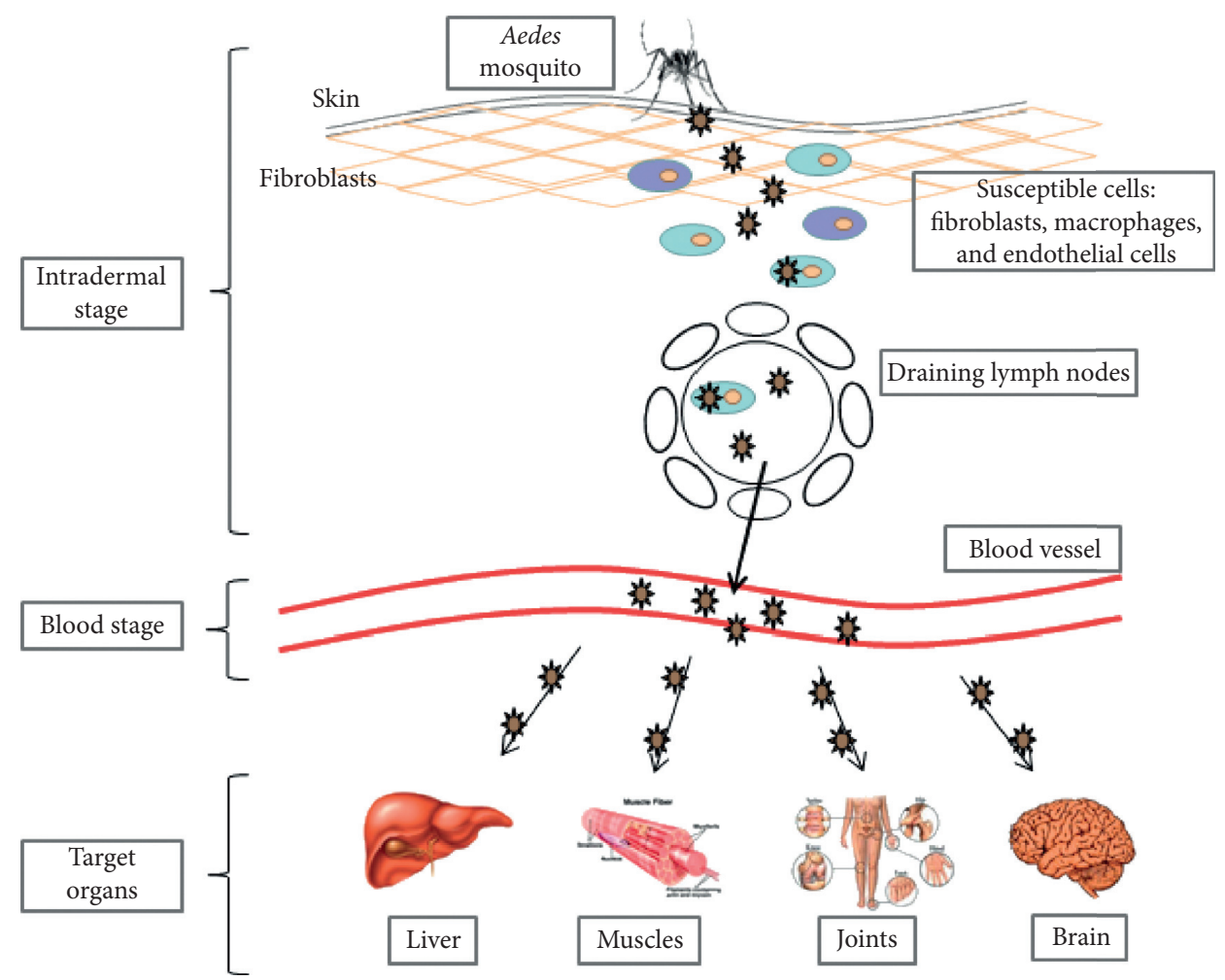

FIGURE 1: Schematic representation of CHIKV dissemination to different tissues and organs [3].

The risk of emergence in Europe is ever increasing due to the increase in "tiger mosquito," Aedes albopictus [6]. First observed in 2004 in the Alpes-Maritimes, the vector was established and active in 33 metropolitan departments in May 2017 [7]. This emergence has made it possible to highlight the need to strengthen the knowledge of health professionals with regard to arboviroses. Therefore, a few mathematical models have been established to describe dynamics of CHIKV viral infection, mostly focusing on virus-to-cell transmission $[8,9]$. However, CHIKV can be spread by cell-to-cell transmission mode [10-13].

In view of this, we will formulate and analyse a generalized within-host CHIKV viral infection model taking into the account both modes of transmission and two discrete delays, in which the first delay $\tau_{1}$ describes the time necessary for the newly produced virions to become mature and infectious and the second delay $\tau_{2}$ represents the time needed to activate the humoral immune response. Then, the model is presented as follows:

$$
\left\{\begin{array}{l}
\dot{U}=s-d_{1} U(t)-f(U(t), I(t), C(t)) C(t)-g(U(t), I(t)) I(t) \\
\dot{I}=f(U(t), I(t), C(t)) C(t)+g(U(t), I(t)) I(t)-d_{2} I(t), \\
\dot{C}=v I\left(t-\tau_{1}\right)-d_{3} C(t)-p A(t) C(t) \\
\dot{A}=\sigma+q A\left(t-\tau_{2}\right) C\left(t-\tau_{2}\right)-d_{4} A(t)
\end{array}\right.
$$

where the general incidence functions $f(U, I, C)$ and $g(U, I)$ assumed to be continuously differentiable satisfy the following hypotheses $[14,15]$ : (i) $\left(H_{0}\right): g(0, I)=0$, for all $I \geq 0$; $(\partial g / \partial U)(U, I) \geq 0$ (or $g(U, I)$ is a monotone increasing function with respect to $T$ when $f \equiv 0)$, and $(\partial g / \partial I)(U, I) \leq 0$, for all $U \geq 0$ and $I \geq 0$

(ii) $\left(H_{1}\right): f(0, I, C)=0$, for all $I \geq 0$ and $C \geq 0$

(iii) $\left(H_{2}\right): f(U, I, C)$ is a monotone increasing function with respect to $U$ (or $(\partial f / \partial U)(U, I, C) \geq 0$ when $g(U, I)$ is a strictly monotone increasing function with respect to $U$ ), for any fixed $I \geq 0$ and $C \geq 0$

(iv) $\left(H_{3}\right): f(U, I, C)$ is a monotone decreasing function with respect to $I$ and $C$

In biological terms, $U(t), I(t), C(t)$, and $A(t)$ indicate the densities of susceptible cells, infected cells, CHIKV particles, and antibodies at time $t$, respectively. The parameter $s$ is the recruitment rate of uninfected cells, and $v$ is the production rate of free CHIKV particles by infected cells. The CHIKV particles are attacked by the antibodies at rate $p A C$. The antibodies are created at rate $\sigma$ and multiplicated at rate $q A C$. The parameters $d_{1}, d_{2}, d_{3}$, and $d_{4}$ are, respectively, the death rates of susceptible cells, infected cells, free CHIKV virions, and antibodies. Moreover, susceptible cells become infected either by free virus at rate $f(U, I, C) C$ or by direct contact with an infected cell at rate $g(U, I) I$. In addition, particular cases of the incidence function $f$ and $g$ are used by Elaiw et al. [16] to model the dynamics of CHIKV with cellular infection and delays. On the other hand, system (1) extends the model presented in [17] when $\tau_{1}=\tau_{2}=0$ and the model proposed in [8] when $f(U, I, C)=\beta_{1} U$ and $g(U, I)=0$. 
The rest of this paper is organized as follows. In Section 2 , we provide some preliminary results concerning the existence, positivity, and boundedness of solutions. Also, we discuss the existence of equilibria. In Section 3, we analyse the stability for the equilibria. We investigate the existence of Hopf bifurcation in Section 4. An application is presented in Section 5. This paper ends with a conclusion in Section 6.

\section{Preliminary Results}

In this section, we first prove the existence, positivity, and boundedness of solutions. After that, we discuss the existence of equilibria.

2.1. Existence, Positivity, and Boundedness of Solutions. According to biological meanings, the initial condition of system (1) is given as follows:

$$
\begin{aligned}
U(\theta) & =\phi_{1}(\theta) \geq 0, \\
I(\theta) & =\phi_{2}(\theta) \geq 0, \\
C(\theta) & =\phi_{3}(\theta) \geq 0, \\
A(\theta) & =\phi_{4}(\theta) \geq 0, \quad \theta \in[-\tau, 0],
\end{aligned}
$$

where $\tau=\max \left\{\tau_{1}, \tau_{2}\right\}$ and $\left(\phi_{1}(\theta), \phi_{2}(\theta), \phi_{3}(\theta), \phi_{4}(\theta)\left(\in \mathscr{C}_{+}=\right.\right.$ $\left.\left.C\left([-\tau, 0], \mathrm{IR}_{+}^{4}\right)\right)\right) . \mathscr{C}_{+}$is the Banach space of continuous functions mapping the interval $[-\tau, 0]$ into $\mathrm{IR}_{+}^{4}$ with the topology of uniform convergence.

It follows from the fundamental theory of functional differential equations [18] that there exists a unique solution of system (1) with initial condition $\left(\phi_{1}, \phi_{2}, \phi_{3}, \phi_{4}\right) \in \mathscr{C}_{+}$.

Next, we investigate the positivity and boundedness of this solution under initial condition (2).

Theorem 1. Under the initial condition (2), the solution of system (1) remains bounded and positive for all $t>0$.

Proof. We first demonstrate that $U(t)>0$ for all $t \geq 0$. By contradiction, we assume that there exists a first time $t_{1}>0$ such that $U\left(t_{1}\right)=0$ and $\dot{U}\left(t_{1}\right) \leq 0$. From the first equation of system (1), we have $\dot{U}\left(t_{1}\right)=s>0$, which leads a contradiction. Then, $U(t)>0$ for all $t \geq 0$. Since $\dot{A}(t)=\eta>0$, and similar to the above, we deduce that $A(t)>0$ for all $t \geq 0$. According to (1), we have

$$
\begin{aligned}
& I(t)=\phi_{2}(0) e^{-d_{2} t \int_{0}^{t} g(U(\theta), I(\theta)) \mathrm{d} \theta}+\int_{0}^{t} f(U(\xi), I(\xi), C(\xi)) C(\xi) e^{-d_{2}(t-\xi)+\int_{0}^{t} g(U(\theta), I(\theta)) \mathrm{d} \theta} \mathrm{d} \xi \\
& C(t)=\phi_{3}(0) e^{-d_{3} t-p \int_{0}^{t} A(\theta) \mathrm{d} \theta}+v \int_{0}^{t} I\left(\xi-\tau_{1}\right) e^{-d_{3}(t-\xi)-\int_{0}^{t} p A(\theta) \mathrm{d} \theta} \mathrm{d} \xi
\end{aligned}
$$

which implies that $I(t)$ and $C(t)$ are nonnegative for all $t \geq 0$.

We consider the following function:

$$
N(t)=U(t)+I(t)+\frac{d_{2}}{2 v} C\left(t+\tau_{1}\right)+\frac{p d_{2}}{2 q v} A\left(t+\tau_{1}+\tau_{2}\right)
$$

Then,

$$
\begin{aligned}
\dot{N}(t)= & s-d_{1} U(t)-\frac{d_{2}}{2} I(t)-\frac{d_{2} d_{3}}{2 v} C\left(t+\tau_{1}\right) \\
& +\frac{p d_{2} \sigma}{2 q v}-\frac{p d_{2} d_{4}}{2 q v} A\left(t+\tau_{1}+\tau_{2}\right) \leq s+\frac{p d_{2} \sigma}{2 q v}-\delta N(t),
\end{aligned}
$$

where $\delta=\min \left\{d_{1},\left(d_{2} / 2\right), d_{3}, d_{4}\right\}$. Hence,

$$
\underset{t \longrightarrow \infty}{\limsup } N(t) \leq \frac{s}{\delta}+\frac{p d_{2} \sigma}{2 q v \delta}
$$

which implies that all solutions of system (1) are bounded. This completes the proof.
2.2. Existence of the Equilibria. Presently, we examine the existence of equilibria. By a basic calculation, system (1) has constantly one infection-free equilibrium of the form $Q_{f}\left(\left(s / d_{1}\right), 0,0,\left(\sigma / d_{4}\right)\right)$. Thus, we characterize the basic reproduction number of our model as follows:

$$
R_{0}=\frac{v f\left(\left(s / d_{1}\right), 0,0\right)+\left(d_{3}+p\left(\sigma / d_{4}\right)\right) g\left(\left(s / d_{1}\right), 0\right)}{d_{2}\left(d_{3}+p\left(\sigma / d_{4}\right)\right)} .
$$

To locate different equilibria of (1), we solve the accompanying system:

$$
\begin{array}{r}
s-d_{1} U-f(U, I, C) C-g(U, I) I=0, \\
f(U, I, C) C+g(U, I) I-d_{2} I=0, \\
v I-d_{3} C-p A C=0, \\
\sigma+q A C-d_{4} A=0 .
\end{array}
$$

From (8)-(11), we obtain $A=\left(\sigma / d_{4}-q C\right), \quad I=\left(\left(d_{3}\right.\right.$ $\left.\left.\left(d_{4}-q C\right)+p \sigma\right) / v\left(d_{4}-q C\right) C\right)=\varphi_{1}(C), \quad U=\left(\left(s-d_{2} \varphi_{1}(C)\right) /\right.$ $\left.d_{1}\right)=\varphi_{2}(C)$, and 


$$
\left.v\left(d_{4}-q C\right) f\left(\varphi_{2}(C), \varphi_{1}(C), C\right)+\left[d_{3}\left(d_{4}-q C\right)+p \sigma\right] g\left(\varphi_{2}(C), \varphi_{1}(C)\right)=d_{2}\left[d_{3}\left(d_{4}-q C\right)+p \sigma\right)\right] .
$$

$A=\sigma / d_{4}-q C \geq 0$ leads to $C<d_{4} / q$. Hence, there is no biological equilibrium when $C \geq d_{4} / q$. Accordingly, we consider the function $\psi$ defined on $\left[0,\left(d_{4} / q\right)\right)$ by

$$
\begin{aligned}
\psi(C)= & v\left(d_{4}-q C\right) f\left(\varphi_{2}(C), \varphi_{1}(C), C\right)+\left[d_{3}\left(d_{4}-q C\right)+p \sigma\right] \\
& \left.\cdot g\left(\varphi_{2}(C), \varphi_{1}(C)\right)-d_{2}\left[d_{3}\left(d_{4}-q C\right)+p \sigma\right)\right] .
\end{aligned}
$$

We have $\varphi_{2}(0)=\left(s / d_{1}\right)>0$ and

$$
\lim _{C \longrightarrow\left(d_{4} / q\right)^{-}} \varphi_{2}(C)=-\infty \text { and } \varphi_{2}^{\prime}(C)=-\frac{d_{2}}{d_{1}} \varphi_{1}^{\prime}(C)<0,
$$

with $\quad \varphi_{1}^{\prime}(C)=\left(\left(d_{3}\left(d_{4}-q C\right)^{2}+p \sigma d_{4}\right) / v\left(d_{4}-q C\right)^{2}\right)>0$. Then, the equation $\varphi_{2}(C)=0$ admits a unique solution $\widetilde{C} \in\left(0,\left(d_{4} / q\right)\right)$. Thus, $\widetilde{A}=\left(\sigma /\left(d_{4}-q \widetilde{C}\right)\right)>0$ and $\psi(\widetilde{C})$ $=-d_{2}\left[d_{3}\left(d_{4}-q \widetilde{C}\right)+p \sigma\right]<0$. Since $\psi(0)=d_{2}\left(d_{3} d_{4}+\right.$ $p \sigma)\left(R_{0}-1\right)>0$ if $R_{0}>1$, we deduce that there exists $C^{*} \in(0, \widetilde{C})$ such that $\psi\left(C^{*}\right)=0$.

From (10) and (11), we find $A^{*}=\left(\sigma /\left(d_{4}-q C^{*}\right)\right)>0$ and $I^{*}=\left(\left(d_{3}+p A^{*}\right) / v\right) C^{*}>0$.

Substitute $C=C^{*}$ and $I=I^{*}$ in (8), and define a function $\varphi_{3}$ as $\varphi_{3}(U)=s-d_{1} U-f\left(U, I^{*}, C^{*}\right) C^{*}-g(U$,
$\left.I^{*}\right) I^{*}$. Due to the fact that $\varphi_{3}(0)=s>0, \varphi_{3}(s / d)=-f((s /$ $\left.\left.d_{1}\right), I^{*}, C^{*}\right) C^{*}-g\left((s / d), I^{*}\right) I^{*}<0$, and $\varphi_{3}$ is a strictly decreasing function of $U$, we deduce that there exists a unique $U^{*} \in\left(0,\left(s / d_{1}\right)\right)$ such that $\varphi_{3}\left(U^{*}\right)=0$. Therefore, model (1) has a unique chronic infection equilibrium $Q^{*}\left(U^{*}, I^{*}\right.$, $C^{*}, A^{*}$ ) when $R_{0}>1$.

The precedent conversations can be summed up in the accompanying outcome.

\section{Theorem 2}

(i) For $R_{0} \leq 1$, model (1) has one infection-free equilibrium $Q_{f}\left(\left(s / d_{1}\right), 0,0,\left(\sigma / d_{4}\right)\right)$

(ii) For $R_{0}>1$, model (1) has a unique chronic infection equilibrium $\quad Q^{*}\left(U^{*}, I^{*}, C^{*}, A^{*}\right) \quad$ with $U^{*} \in\left(0,\left(s / d_{1}\right)\right), I^{*}>0, C^{*}>0$, and $A^{*}>0$

\section{Stability Analysis of Equilibria}

In this section, we concentrate on the stability of infectionfree equilibrium of system (1). The characteristic equation of system (1) is noted as

$$
\left|\begin{array}{cccc}
-d_{1}-C \frac{\partial f}{\partial U}-I \frac{\partial g}{\partial U}-\xi & -C \frac{\partial f}{\partial I}-I \frac{\partial g}{\partial I}-g(U, I) & -C \frac{\partial f}{\partial C}-f(U, I, C) & 0 \\
C \frac{\partial f}{\partial U}+I \frac{\partial g}{\partial U} & C \frac{\partial f}{\partial I}+I \frac{\partial g}{\partial I}+g(U, I)-d_{2}-\xi & C \frac{\partial f}{\partial C}+f(U, I, C) & 0 \\
0 & v e^{-\xi \tau_{1}} & -d_{3}-p A-\xi & -p C \\
0 & 0 & q A e^{-\xi \tau_{2}} & q C e^{-\xi \tau_{2}}-d_{4}-\xi
\end{array}\right|=0 .
$$

First, we have the following result.

Proof. Examining (15) at $Q_{f}$, we obtain

Theorem 3. For any $\tau_{2}$ and $\tau_{1}=0$, the infection-free equilibrium $Q_{f}$ is locally asymptotically stable if $R_{0}<1$ and becomes unstable if $R_{0}>1$.

$$
\left(\xi+d_{1}\right)\left(\xi+d_{4}\right)\left(\begin{array}{c}
\xi^{2}+\left[d_{3}+d_{2}+p \frac{\sigma}{d_{4}}-g\left(\frac{s}{d_{1}}, 0\right)\right] \xi-v e^{-\xi \tau_{1}} f\left(\frac{s}{d_{1}}, 0,0\right) \\
-\left(d_{3}+p \frac{\sigma}{d_{4}}\right)\left(g\left(\frac{s}{d_{1}}, 0\right)-d_{2}\right)
\end{array}\right)=0 .
$$

When $\tau_{1}=0$, from equation (16), we obtain

$$
\left(\xi+d_{1}\right)\left(\xi+d_{4}\right)\left(\xi^{2}+\left[d_{3}+d_{2}+p \frac{\sigma}{d_{4}}-g\left(\frac{s}{d_{1}}, 0\right)\right] \xi+d_{2}\left(d_{3}+p \frac{\sigma}{d_{4}}\right)\left(1-R_{0}\right)\right)=0
$$


Therefore, the roots of this equation are

$$
\begin{aligned}
& \xi_{1}=-d_{1}, \\
& \xi_{2}=-d_{4}, \\
& \xi_{3}=\frac{-\left(d_{2}+d_{3}+p\left(\sigma / d_{4}\right)-g\left(s / d_{1}, 0\right)\right)-\sqrt{\Delta}}{2}, \\
& \xi_{4}=\frac{-\left(d_{2}+d_{3}+p \sigma / d_{4}-g\left(s / d_{1}, 0\right)\right)+\sqrt{\Delta}}{2},
\end{aligned}
$$

of which $\quad \Delta=\left(d_{2}+d_{3}+p \sigma / d_{4}-g\left(s / d_{1}, 0\right)\right)^{2}-4 d_{2}$ $\left(d_{3}+p \sigma / d_{4}\right)\left(1-R_{0}\right)$. Obviously, $\xi_{1}, \xi_{2}$, and $\xi_{3}$ are negative. Furthermore, $\xi_{4}$ is negative if $R_{0}<1$ and positive if $R_{0}>1$. Consequently, $Q_{f}$ is locally asymptotically stable if $R_{0}<1$ and unstable if $R_{0}>1$.

The following theorem characterizes the global stability of the infection-free equilibrium $Q_{f}$ when $R_{0} \leq 1$.

Theorem 4. For any $\tau_{1}$ and $\tau_{2}$, the infection-free equilibrium $Q_{f}$ is globally asymptotically stable if $R_{0} \leq 1$.

Proof. We establish a Lyapunov function as follows:

$$
L(t)=I(t)+\frac{f\left(\left(s / d_{1}\right), 0,0\right)}{d_{3}+p\left(\sigma / d_{4}\right)} C(t)+\frac{v f\left(\left(s / d_{1}\right), 0,0\right)}{d_{3}+p\left(\sigma / d_{4}\right)} \int_{t-\tau_{1}}^{t} I(\theta) \mathrm{d} \theta .
$$

Computing the time derivative of $L$ along the solutions of (1), we find

$$
\begin{aligned}
\frac{\mathrm{d} L}{\mathrm{~d} t}= & \left(f(U, I, C)-\frac{d_{3}+p A}{d_{3}+p\left(\sigma / d_{4}\right)} f\left(\frac{s}{d_{1}}, 0,0\right)\right) C \\
& +d_{2}\left(\frac{v f\left(s / d_{1}, 0,0\right)+\left(d_{2}+p\left(\sigma / d_{4}\right)\right) g(U, I)}{d_{2}\left(d_{3}+p\left(\sigma / d_{4}\right)\right)}-1\right) I \\
\leq & \left(f(U, 0,0)-f\left(\frac{s}{d_{1}}, 0,0\right)\right) C+a\left(R_{0}-1\right) I \leq a\left(R_{0}-1\right) I .
\end{aligned}
$$

Given that $R_{0} \leq 1$, we have $(\mathrm{d} L / \mathrm{d} t) \leq 0$. Likewise, it is not difficult to show that the largest invariant set in $\{(U, I, C, A) \mid(\mathrm{d} L / \mathrm{d} t)=0\}$ is $\left\{Q_{f}\right\}$. By the LaSalle's invariance principle [19], $Q_{f}$ is globally asymptotically stable for $R_{0} \leq 1$.

Next, we focus on the global stability of the chronic infection equilibrium $Q^{*}$ by assuming that $R_{0}>1$, and for all $U, I, C>0$, we consider the following hypothesis:

$$
\begin{array}{r}
\left(1-\frac{f(U, I, C)}{f\left(U, I^{*}, C^{*}\right)}\right)\left(\frac{f\left(U, I^{*}, C^{*}\right)}{f(U, I, C)}-\frac{C}{C^{*}}\right) \leq 0, \\
\left(1-\frac{f\left(U^{*}, I^{*}, C^{*}\right) g(U, I)}{f\left(U, I^{*}, C^{*}\right) g\left(U^{*}, I^{*}\right)}\right)\left(\frac{f\left(U, I^{*}, C^{*}\right) g\left(U^{*}, I^{*}\right)}{f\left(U^{*}, I^{*}, C^{*}\right) g(U, I)}-\frac{I}{I^{*}}\right) \leq 0 .
\end{array}
$$

Theorem 5. Assume that (21) holds. For any $\tau_{1}$, if $\tau_{2}=0$ and $R_{0}>1$, then the chronic infection equilibrium $Q^{*}$ is globally

Proof. Consider the following Lyapunov function: asymptotically stable.

$$
\begin{aligned}
W(t)= & U(t)-U^{*}-\int_{U^{*}}^{U} \frac{f\left(U^{*}, I^{*}, C^{*}\right)}{f\left(X, I^{*}, C^{*}\right)} \mathrm{d} X+I^{*} \Phi\left(\frac{I(t)}{I^{*}}\right)+\frac{f\left(U^{*}, I^{*}, C^{*}\right) C^{*}}{v I^{*}} C^{*} \Phi\left(\frac{C(t)}{C^{*}}\right) \\
& +\frac{q f\left(U^{*}, I^{*}, C^{*}\right) C^{*}}{q v I^{*}} A^{*} \Phi\left(\frac{A(t)}{A^{*}}\right)+f\left(U^{*}, I^{*}, C^{*}\right) C^{*} \int_{t-\tau_{1}}^{t} \Phi\left(\frac{I(\theta)}{I^{*}}\right) \mathrm{d} \theta,
\end{aligned}
$$

where $\Phi(x)=x-1-\ln x, x>0$. Thus, the time derivative of $W$ along the positive solutions of (1) satisfies

$$
\begin{aligned}
\frac{\mathrm{d} W}{\mathrm{~d} t}= & \left(1-\frac{f\left(U^{*}, I^{*}, C^{*}\right)}{f\left(U, I^{*}, C^{*}\right)}\right) \dot{U}+\left(1-\frac{I^{*}}{I}\right) \dot{I}+\frac{f\left(U^{*}, I^{*}, C^{*}\right) C^{*}}{v I^{*}}\left(1-\frac{C^{*}}{C}\right) \dot{C} \\
& \frac{p f\left(U^{*}, I^{*}, C^{*}\right) C^{*}}{q v I^{*}}\left(1-\frac{A^{*}}{A}\right) \dot{A}+f\left(U^{*}, I^{*}, C^{*}\right) C^{*} \frac{d}{d t} \int_{t-\tau_{1}}^{t} \Phi\left(\frac{I(\theta)}{I^{*}}\right) \mathrm{d} \theta,
\end{aligned}
$$


where $(\mathrm{d} / \mathrm{d} t) \int_{t-\tau_{1}}^{t} \Phi\left(I(\theta) / I^{*}\right) \mathrm{d} \theta=\left(I-I\left(t-\tau_{1}\right) / I^{*}\right)$

$+\ln \left(I\left(t-\tau_{1}\right) / I\right)$. Therefore, we have

$$
\begin{aligned}
\frac{\mathrm{d} W}{\mathrm{~d} t}= & \left(1-\frac{f\left(U^{*}, I^{*}, C^{*}\right)}{f\left(U, I^{*}, C^{*}\right)}\right)\left(s-d_{1} U-f(U, I, C) C-g(U, I) I\right) \\
& +\left(1-\frac{I^{*}}{I}\right)\left(f(U, I, C) C+g(U, I) I-d_{2} I\right)+\frac{f\left(U^{*}, I^{*}, C^{*}\right) C^{*}}{v I^{*}}\left(1-\frac{C^{*}}{C}\right)\left(v I\left(t-\tau_{1}\right)-d_{3} C-p A C\right) \\
& +\frac{p f\left(U^{*}, I^{*}, C^{*}\right) C^{*}}{q v I^{*}}\left(1-\frac{A^{*}}{A}\right)\left(\sigma+q A C-d_{4} A\right)+f\left(U^{*}, I^{*}, C^{*}\right) C^{*}\left(\frac{I-I\left(t-\tau_{1}\right)}{I^{*}}+\ln \frac{I\left(t-\tau_{1}\right)}{I}\right) .
\end{aligned}
$$

Substituting $s=d_{1} U^{*}+f\left(U^{*}, I^{*}, C^{*}\right) C^{*}+g\left(U^{*}, I^{*}\right) I^{*}$,

$v I^{*}=d_{3} C^{*}+q A^{*} C^{*}$, and $\sigma=d_{4} A^{*}-q A^{*} C^{*}$, we obtain

$$
\begin{aligned}
\frac{\mathrm{d} W}{\mathrm{~d} t}= & d_{1} U^{*}\left(1-\frac{U}{U^{*}}\right)\left(1-\frac{f\left(U^{*}, I^{*}, C^{*}\right)}{f\left(U, I^{*}, C^{*}\right)}\right)+f\left(U^{*}, I^{*}, C^{*}\right) \\
& C^{*}\left(-1-\frac{C}{C^{*}}+\frac{f(U, I, C) C}{f\left(U, I^{*}, C^{*}\right) C^{*}}+\frac{f\left(U, I^{*}, C^{*}\right)}{f(U, I, C)}\right)+f\left(U^{*}, I^{*}, C^{*}\right) C^{*} \\
& \left(4-\frac{f\left(U^{*}, I^{*}, C^{*}\right)}{f\left(U, I^{*}, C^{*}\right)}-\frac{f\left(U, I^{*}, C^{*}\right)}{f(U, I, C)}-\frac{f(U, I, C) C I^{*}}{f\left(U^{*}, I^{*}, C^{*}\right) C^{*} I}-\frac{C^{*} I\left(t-\tau_{1}\right)}{C I^{*}}+\ln \frac{I\left(t-\tau_{1}\right)}{I}\right) \\
& +g\left(U^{*}, I^{*}\right) I^{*}\left(-1-\frac{I}{I^{*}}-\frac{f\left(U^{*}, I^{*}, C^{*}\right) g(U, I) I}{f\left(U, I^{*}, C^{*}\right) g\left(U^{*}, I^{*}\right) I^{*}}+\frac{f\left(U, I^{*}, C^{*}\right) g\left(U^{*}, I^{*}\right)}{f\left(U^{*}, I^{*}, C^{*}\right) g(U, I)}\right) \\
& +g\left(U^{*}, I^{*}\right) I^{*}\left(3-\frac{f\left(U^{*}, I^{*}, C^{*}\right)}{f\left(U, I^{*}, C^{*}\right)}-\frac{f\left(U, I^{*}, C^{*}\right) g\left(U^{*}, I^{*}\right)}{f\left(U^{*}, I^{*}, C^{*}\right) g(U, I)}-\frac{g(U, I)}{g\left(U^{*}, I^{*}\right)}\right) \\
& -\frac{p \sigma f\left(U^{*}, I^{*}, C^{*}\right) C^{*}}{q v I^{*} A^{*} A}\left(A-A^{*}\right)^{2} .
\end{aligned}
$$

Thus,

$$
\begin{aligned}
\frac{\mathrm{d} W}{\mathrm{~d} t}= & d_{1} U^{*}\left(1-\frac{U}{U^{*}}\right)\left(1-\frac{f\left(U^{*}, I^{*}, C^{*}\right)}{f\left(U, I^{*}, C^{*}\right)}\right) \\
& +f\left(U^{*}, I^{*}, C^{*}\right) C^{*}\left(-1-\frac{C}{C^{*}}+\frac{f(U, I, C) C}{f\left(U, I^{*}, C^{*}\right) C^{*}}+\frac{f\left(U, I^{*}, C^{*}\right)}{f(U, I, C)}\right) \\
& +g\left(U^{*}, I^{*}\right) I^{*}\left(-1-\frac{I}{I^{*}}-\frac{f\left(U^{*}, I^{*}, C^{*}\right) g(U, I) I}{f\left(U, I^{*}, C^{*}\right) g\left(U^{*}, I^{*}\right) I^{*}}+\frac{f\left(U, I^{*}, C^{*}\right) g\left(U^{*}, I^{*}\right)}{f\left(U^{*}, I^{*}, C^{*}\right) g(U, I)}\right) \\
& -f\left(U^{*}, I^{*}, C^{*}\right) C^{*}\left[\Phi\left(\frac{f\left(U^{*}, I^{*}, C^{*}\right)}{f\left(U, I^{*}, C^{*}\right)}\right)+\Phi\left(\frac{f\left(U, I^{*}, C^{*}\right)}{f(U, I, C)}\right)+\Phi\left(\frac{C^{*} I\left(t-\tau_{1}\right)}{C I^{*}}\right)+\Phi\left(\frac{f(U, I, C) C I^{*}}{f\left(U^{*}, I^{*}, C^{*}\right) C^{*} I}\right)\right] \\
& -g\left(U^{*}, I^{*}\right) I^{*}\left[\Phi\left(\frac{f\left(U^{*}, I^{*}, C^{*}\right)}{f\left(U, I^{*}, C^{*}\right)}\right)+\Phi\left(\frac{f\left(U, I^{*}, C^{*}\right) g\left(U^{*}, I^{*}\right)}{f\left(U^{*}, I^{*}, C^{*}\right) g(U, I)}\right)+\Phi\left(\frac{g(U, I)}{g\left(U^{*}, I^{*}\right)}\right)\right] \\
& -\frac{p \sigma f\left(U^{*}, I^{*}, C^{*}\right) C^{*}}{q v I^{*} A^{*} A}\left(A-A^{*}\right)^{2} .
\end{aligned}
$$


By $\left(H_{2}\right)$, we find that

$$
\left(1-\frac{U}{U^{*}}\right)\left(1-\frac{f\left(U^{*}, I^{*}, C^{*}\right)}{f\left(U, I^{*}, C^{*}\right)}\right) \leq 0 .
$$

By (21), we obtain

$$
\begin{aligned}
-1-\frac{C}{C^{*}}+\frac{f(U, I, C) C}{f\left(U, I^{*}, C^{*}\right) C^{*}}+\frac{f\left(U, I^{*}, C^{*}\right)}{f(U, I, C)}= & \left(1-\frac{f(U, I, C)}{f\left(U, I^{*}, C^{*}\right)}\right)\left(\frac{f\left(U, I^{*}, C^{*}\right)}{f(U, I, C)}-\frac{C}{C^{*}}\right) \leq 0 \\
& -1-\frac{I}{I^{*}}-\frac{f\left(U^{*}, I^{*}, C^{*}\right) g(U, I) I}{f\left(U, I^{*}, C^{*}\right) g\left(U^{*}, I^{*}\right) I^{*}}+\frac{f\left(U, I^{*}, C^{*}\right) g\left(U^{*}, I^{*}\right)}{f\left(U^{*}, I^{*}, C^{*}\right) g(U, I)} \\
= & \left(1-\frac{f\left(U^{*}, I^{*}, C^{*}\right) g(U, I)}{f\left(U, I^{*}, C^{*}\right) g\left(U^{*}, I^{*}\right)}\right)\left(\frac{f\left(U, I^{*}, C^{*}\right) g\left(U^{*}, I^{*}\right)}{f\left(U^{*}, I^{*}, C^{*}\right) g(U, I)}-\frac{I}{I^{*}}\right) \leq 0 .
\end{aligned}
$$

Since $\Phi(x) \geq 0$, we have $(\mathrm{d} W / \mathrm{d} t) \leq 0$ with equality if and only if $U=U^{*}, I=I^{*}, C=C^{*}$, and $A=A^{*}$. From LaSalle's invariance principle, we deduce that the chronic infection equilibrium $Q^{*}$ is globally asymptotically stable when $R_{0}>1$.

\section{Hopf Bifurcation Analysis}

In this section, we investigate the bifurcation at the infection equilibrium $Q^{*}$. By computing the characteristic equation for system (1) at $Q^{*}$, we find

$$
\begin{aligned}
\xi^{4}+ & a_{1} \xi^{3}+a_{2} \xi^{2}+a_{3} \xi+a_{4}+\left(b_{1} \xi^{3}+b_{2} \xi^{2}+b_{3} \xi+b_{4}\right) e^{-\xi \tau_{2}} \\
& +\left(c_{1} \xi^{2}+c_{2} \xi+c_{3}\right) e^{-\xi \tau_{1}}+\left(r_{1} \xi+r_{2}\right) e^{-\xi\left(\tau_{1}+\tau_{2}\right)}=0
\end{aligned}
$$

where

$$
\begin{aligned}
& a_{1}=d_{1}+d_{3}+d_{4}+M+A^{*}-N \\
& a_{2}=\left(d_{1}+M-N\right)\left(d_{3}+d_{4}+p A^{*}\right)+d_{4}\left(d_{3}+p A^{*}\right)+d_{1}\left(d_{2}-N\right)+M d_{2}, \\
& a_{3}=d_{1}\left(d_{2}-N\right)\left(d_{3}+d_{4}+p A^{*}\right)+\left(d_{3}+p A^{*}\right)\left[d_{4}\left(d_{1}+M-N\right)+M d_{2}\right]+M d_{2} d_{4}, \\
& a_{4}=d_{4}\left(d_{3}+p A^{*}\right)\left[d_{1}\left(d_{2}-N\right)+M d_{2}\right] \\
& b_{1}=-q C^{*} \\
& b_{2}=-q C^{*}\left(d_{1}+d_{3}+M-N\right) \\
& b_{3}=-q C^{*}\left[d_{2}\left(M-p A^{*}\right)+d_{1}\left(d_{2}-N\right)+d_{3}\left(d_{1}+M-N\right)\right] \\
& b_{4}=-q C^{*}\left[p A^{*}\left(N d_{1}-M d_{2}+\left(d_{3}+p A^{*}\right)\left[d_{1}\left(d_{2}-N\right)+M d_{2}\right]\right]\right. \\
& c_{1}=-v Q \\
& c_{2}=-v Q\left(d_{4}+1\right) \\
& c_{3}=-v Q d_{4} \\
& r_{1}=q C^{*} v \\
& r_{2}=q C^{*} v d_{1}
\end{aligned}
$$

with $\quad M=C^{*}(\partial f / \partial U)\left(U^{*}, I^{*}, C^{*}\right)+I^{*}(\partial g / \partial U)\left(U^{*}, I^{*}\right)$, $Q=C^{*}(\partial f / \partial C)\left(U^{*}, I^{*}, C^{*}\right)+f\left(U^{*}, I^{*}, C^{*}\right)$, and $N=C^{*}$ $(\partial f / \partial I)\left(U^{*}, I^{*}, C^{*}\right)+I^{*}(\partial g / \partial I)\left(U^{*}, I^{*}\right)+g\left(U^{*}, I^{*}\right)$.
However, when $\tau_{1}>0$, equation (29) is too complicated. Therefore, in the following discussions, we assume that $\tau_{1}=$ 0 and $\tau_{2}>0$. Then, equation (29) is diminished to

$$
\xi^{4}+H_{1} \xi^{3}+H_{2} \xi^{2}+H_{3} \xi+H_{4}+\left(I_{1} \xi^{3}+I_{2} \xi^{2}+I_{3} \xi+I_{4}\right) e^{-\xi \tau_{2}}=0
$$


where

$$
\begin{aligned}
H_{1}= & a_{1}=d_{1}+d_{3}+d_{4}+M+A^{*}-N, \\
H_{2}= & a_{2}+c_{1}=\left(d_{1}+M-N\right)\left(d_{3}+d_{4}+p A^{*}\right)+d_{4}\left(d_{3}+p A^{*}\right)+d_{1}\left(d_{2}-N\right)+M d_{2}-v Q, \\
H_{3}= & a_{3}+c_{2}=d_{1}\left(d_{2}-N\right)\left(d_{3}+d_{4}+p A^{*}\right)+\left(d_{3}+p A^{*}\right)\left[d_{4}\left(d_{1}+M-N\right)+M d_{2}\right] \\
& +M d_{2} d_{4}-v Q\left(d_{4}+1\right), \\
H_{4}= & a_{4}+c_{3}=d_{4}\left(d_{3}+p A^{*}\right)\left[d_{1}\left(d_{2}-N\right)+M d_{2}\right]-v Q d_{4}, \\
I_{1}= & b_{1}=-q C^{*} \\
I_{2}= & b_{2}=-q C^{*}\left(d_{1}+d_{3}+M-N\right), \\
I_{3}= & b_{3}+r_{1}=-q C^{*}\left[d_{2}\left(M-p A^{*}\right)+d_{1}\left(d_{2}-N\right)+d_{3}\left(d_{1}+M-N\right)-v\right], \\
I_{4}= & b_{4}+r_{2}=-q C^{*}\left[p A^{*}\left(N d_{1}-M d_{2}+\left(d_{3}+p A^{*}\right)\left[d_{1}\left(d_{2}-N\right)+M d_{2}\right]-v d_{1}\right] .\right.
\end{aligned}
$$

Let $\xi=i \omega(\omega>0)$ be a purely imaginary root of (31). Separating real and imaginary parts, it follows that

$$
\left\{\begin{array}{l}
\omega^{4}-H_{2} \omega^{2}+H_{4}=\left(I_{2} \omega^{2}-I_{4}\right) \cos \left(\tau_{2} \omega\right)+\left(I_{1} \omega^{3}-I_{3} \omega\right) \sin \left(\tau_{2} \omega\right), \\
-H_{1} \omega^{3}+H_{3} \omega=\left(I_{1} \omega^{3}-I_{3} \omega\right) \cos \left(\tau_{2} \omega\right)-\left(I_{2} \omega^{2}-I_{4}\right) \sin \left(\tau_{2} \omega\right) .
\end{array}\right.
$$

Squaring and adding the two equations of (33), it follows that

$$
\omega^{8}+L_{1} \omega^{6}+L_{2} \omega^{4}+L_{3} \omega^{2}+L_{4}=0
$$

where

$$
\begin{aligned}
& L_{1}=H_{1}^{2}-2 H_{2}-I_{1}^{2}, \\
& L_{2}=H_{2}^{2}-2 H_{1} H_{3}+2 H_{4}+2 I_{1} I_{3}-I_{2}^{2}, \\
& L_{3}=H_{3}^{2}-2 H_{2} H_{4}+2 I_{2} I_{4}-I_{3}^{2}, \\
& L_{4}=H_{4}^{2}-I_{4}^{2} .
\end{aligned}
$$

Let $z=\omega^{2}$, then equation (34) becomes

$$
\Psi(z):=z^{4}+L_{1} z^{3}+L_{2} z^{2}+L_{3} z+L_{4}=0 .
$$

It is clear that when $L_{4}<0$, equation (36) has at least one positive root because $\Psi(0)=L_{4}<0$ and $\lim _{z \longrightarrow+\infty} \Psi(z)=$ $+\infty$. Moreover, we obtain

$$
\Psi^{\prime}(z)=4 z^{3}+3 L_{1} z^{2}+2 L_{2} z+L_{3} .
$$

Denote that

$$
\begin{aligned}
& \gamma_{1}=\frac{8 L_{2}-3 L_{1}^{2}}{16}, \\
& \delta_{1}=\frac{L_{1}^{3}-4 L_{1} L_{2}+8 L_{2}}{32}, \\
& \Delta=\left(\frac{\gamma_{1}}{2}\right)^{3}+\left(\frac{\delta_{1}}{2}\right)^{3}, \\
& j=e^{i(2 \pi / 3)}=-\frac{1}{2}+i \frac{\sqrt{3}}{2} .
\end{aligned}
$$

Applying the Cardano formula, the cubic equation (37) has the following roots:

$$
\begin{aligned}
& v_{1}=\sqrt[3]{-\frac{\delta_{1}}{2}+\sqrt{\Delta}}+\sqrt[3]{-\frac{\delta_{1}}{2}+\sqrt{\Delta}}-\frac{L_{1}}{4}, \\
& v_{2}=j \sqrt[3]{-\frac{\delta_{1}}{2}+\sqrt{\Delta}}+j \sqrt[3]{-\frac{\delta_{1}}{2}+\sqrt{\Delta}}-\frac{L_{1}}{4}, \\
& v_{3}=j^{2} \sqrt[3]{-\frac{\delta_{1}}{2}+\sqrt{\Delta}}+j \sqrt[3]{-\frac{\delta_{1}}{2}+\sqrt{\Delta}}-\frac{L_{1}}{4} .
\end{aligned}
$$

When $\Delta>0$, the first root $\nu_{1}$ is a real number and the other two, $v_{2}$ and $v_{3}$, are conjugate complex numbers. In this situation,

$$
\Psi^{\prime}(z)=4\left(z-v_{1}\right)\left(z^{2}-2 \operatorname{Re}\left(v_{2}\right) z+\left|v_{2}\right|^{2}\right) .
$$

We assume that $\Psi(z)$ is a decreasing function on the interval $\left(-\infty, v_{1}\right]$ and increasing function on $\left[v_{1},+\infty\right)$. Since $z^{2}-2 \operatorname{Re}\left(\nu_{2}\right) z+\left|\nu_{2}\right|^{2}>0$ for all $z \in \mathbb{R}$, it attains its strict global minimum at $z=v_{1}$.

When $\Delta=0$, all roots are real with $v_{1}=\left(3 \gamma_{1} / \delta_{1}\right)-\left(L_{1} / 4\right)$ and $\nu_{2}=v_{3}=\left(3 \gamma_{1} / 2 \delta_{1}\right)-\left(L_{1} / 4\right)$. Then,

$$
\Psi^{\prime}(z)=4\left(z-v_{1}\right)\left(z-v_{2}\right)^{2} .
$$

Hence, $\Psi(z)$ is a decreasing function on $\left(-\infty, v_{1}\right]$ and increasing function on $\left[v_{1},+\infty\right)$. Also, it attains its strict global minimum at $z=v_{1}$. Consequently, if $L_{4} \geq 0$ and $\Delta \geq 0$, then equation (36) has a positive root if and only if $v_{1}>0$ and $\Psi\left(v_{1}\right) \leq 0$.

When $\Delta<0$, all three roots are real and distinct. In this case, $\Psi^{\prime}(z)$ can be changed as

$$
\Psi^{\prime}(z)=4\left(z-v_{1}\right)\left(z-v_{2}\right)\left(z-v_{3}\right) .
$$

Similarly, we obtain that if $L_{4} \geq 0$ and $\Delta<0$, then equation (36) has a positive root if and only if there exists at least one $\nu^{*} \in\left\{v_{1}, v_{2}, v_{3}\right\}$ such that $\nu^{*}>0$ and $\Psi\left(\nu^{*}\right) \leq 0$. lemma.

Outlining the above discussions, we obtain the following 
Lemma 1. For the polynomial equation (36), the following results are true:

(i) If $L_{4}<0$, then equation (36) has at least one positive root

(ii) If $L_{4} \geq 0$ and $\Delta \geq 0$, then equation (36) has positive root if and only if $\nu_{1}>0$ and $\Psi\left(\nu_{1}\right) \leq 0$

(iii) If $L_{4} \geq 0$ and $\Delta<0$, then equation (36) has a positive root if and only if there exists at least one $\nu^{*} \in\left\{\nu_{1}, \nu_{2}, v_{3}\right\}$ such that $\nu^{*}>0$ and $\Psi\left(\nu^{*}\right) \leq 0$

In light of this lemma, we acknowledge the following conditions:

(a) $L_{4}<0$

(b) $L_{4} \geq 0, \Delta \geq 0, v_{1}>0$, and $\Psi\left(v_{1}\right) \leq 0$ (c) $L_{4} \geq 0, \quad \Delta<0$, and there exists at least one $v^{*} \in\left\{\nu_{1}, v_{2}, v_{3}\right\}$ such that $v^{*}>0$ and $\Psi\left(\nu^{*}\right) \leq 0$

In the case that the conditions $(a)-(c)$ are not fulfilled, then equation (14) has no positive roots. Consequently, the infection equilibrium $Q^{*}$ is locally asymptotically stable for all delay $\tau_{2} \geq 0$. As a result, the existence of Hopf bifurcation is preposterous.

Presently, we expect that one of the conditions, $(a)-(c)$, is fulfilled. We suppose that equation (36) has $k_{0}$ positive roots, where $k_{0} \in\{1,2,3,4\}$. Denote the positive root of (36) by $z_{k}, k=1,2, \ldots, k_{0}$. Then, equation (34) has positive roots $\omega_{k}=\sqrt{z_{k}}$. In accordance with (33), we obtain

$$
\begin{aligned}
& \cos \left(\omega_{k} \tau_{2}\right)=\frac{\left(\omega_{k}^{4}-H_{2} \omega_{k}^{2}+H_{4}\right)\left(I_{2} \omega_{k}^{2}-I_{4}\right)+\left(-H_{1} \omega_{k}^{3}+H_{3} \omega_{k}\right)\left(I_{1} \omega_{k}^{3}-I_{3} \omega_{k}\right)}{\left(I_{2} \omega_{k}^{2}-I_{4}\right)^{2}+\left(I_{1} \omega_{k}^{3}-I_{3} \omega_{k}\right)^{2}}=\Upsilon_{1}\left(\omega_{k}\right), \\
& \sin \left(\omega_{k} \tau_{2}\right)=\frac{\left(\omega_{k}^{4}-H_{2} \omega_{k}^{2}+H_{4}\right)\left(I_{1} \omega_{k}^{3}-I_{3} \omega_{k}\right)-\left(-H_{1} \omega_{k}^{3}+H_{3} \omega_{k}\right)\left(I_{2} \omega_{k}^{2}-I_{4}\right)}{\left(I_{2} \omega_{k}^{2}-I_{4}\right)^{2}+\left(I_{1} \omega_{k}^{3}-I_{3} \omega_{k}\right)^{2}}=\Upsilon_{2}\left(\omega_{k}\right) .
\end{aligned}
$$

Define

$$
\tau_{2, k}^{(n)}= \begin{cases}\frac{1}{\omega_{k}}\left[\arccos \left(\Upsilon_{1}\left(\omega_{k}\right)\right)+2 n \pi\right], & \text { If } Y_{2}\left(\omega_{k}\right) \geq 0, \\ \frac{1}{\omega_{k}}\left[2 \pi-\arccos \left(\Upsilon_{1}\left(\omega_{k}\right)\right)+2 n \pi\right], & \text { If } \Upsilon_{2}\left(\omega_{k}\right)<0,\end{cases}
$$

where $k=1,2, \ldots, k_{0}$ and $n \in \mathbb{N}$. Hence,

$$
\begin{gathered}
\tau_{0}=\tau_{2, k_{0}}^{(0)}=\min _{1 \leq k<k_{0}}\left\{\tau_{2, k}^{(0)}\right\}, \\
\omega_{0}=\omega_{k_{0}} \text { and } z_{0}=z_{k_{0}} .
\end{gathered}
$$

Let $\xi(\tau)=\alpha(\tau)+i \omega(\tau)$ be the root of equation (31) at $\tau=\tau_{0}$ satisfying $\alpha\left(\tau_{0}\right)=0$ and $\omega\left(\tau_{0}\right)=\omega_{0}$. Then, we obtain the following result.

Lemma 2. If $\Psi^{\prime}\left(\nu_{0}\right) \neq 0$, then $d \operatorname{Re} \xi\left(\tau_{2}\right) /\left.d \tau_{2}\right|_{\tau_{2}=\tau_{0}}$ and $\Psi^{\prime}\left(\tau_{2}\right)$ have the same sign.

Proof. Differentiating both sides of equation (31) with respect to $\tau_{2}$ and noticing that $\xi$ is a function of $\tau$ yield

$$
\begin{aligned}
\left(\frac{\mathrm{d} \xi}{\mathrm{d} \tau_{2}}\right)^{-1}= & \frac{4 \xi^{3}+3 H_{1} \xi^{2}+2 H_{2} \xi+H_{3}}{-\xi e^{-\xi \tau_{2}}\left(I_{1} \xi^{3}+I_{2} \xi^{2}+I_{3} \xi+I_{4}\right)}+\frac{3 I_{1} \xi^{2}+2 I_{2} \xi+I_{3}}{\xi I_{1} \xi^{3}+I_{2} \xi^{2}+I_{3} \xi+I_{4}}-\frac{\tau_{2}}{\xi} \\
= & \frac{4 \xi^{3}+3 H_{1} \xi^{2}+2 H_{2} \xi+H_{3}}{-\xi\left(\xi^{4}+H_{1} \xi^{3}+H_{2} \xi^{2}+H_{3} \xi+H_{4}\right)}+\frac{3 I_{1} \xi^{2}+2 I_{2} \xi+I_{3}}{\xi\left(I_{1} \xi^{3}+I_{2} \xi^{2}+I_{3} \xi+I_{4}\right)}-\frac{\tau_{2}}{\xi} \\
& \left(4 \xi^{3}+3 H_{1} \xi^{2}+2 H_{2} \xi+H_{3}\right) \frac{\mathrm{d} \xi}{\mathrm{d} \tau_{2}}+e^{-\xi \tau_{2}}\left(3 I_{1} \xi^{2}+2 I_{2} \xi+I_{3}\right) \frac{\mathrm{d} \xi}{\mathrm{d} \tau_{2}} \\
& -e^{-\xi \tau_{2}}\left(I_{1} \xi^{3}+I_{2} \xi^{2}+I_{3} \xi+I_{4}\right)\left(\tau_{2} \frac{\mathrm{d} \xi}{\mathrm{d} \tau_{2}}+\xi\right)=0 .
\end{aligned}
$$


The fact that

leads to

$$
\operatorname{sign}\left[\left.\frac{\mathrm{d} \operatorname{Re} \xi\left(\tau_{2}\right)}{\mathrm{d} \tau_{2}}\right|_{\tau_{2}=\tau_{0}}\right]=\operatorname{sign}\left[\left.\operatorname{Re}\left(\frac{\mathrm{d} \xi\left(\tau_{2}\right)}{\mathrm{d} \tau_{2}}\right)^{-1}\right|_{\tau_{2}=\tau_{0}}\right],
$$

$$
\begin{aligned}
\operatorname{sign}\left[\left.\frac{\mathrm{dRe} \xi\left(\tau_{2}\right)}{\mathrm{d} \tau_{2}}\right|_{\tau_{2}=\tau_{0}}\right]= & \operatorname{sign}\left[\left.\operatorname{Re}\left[\frac{4 \xi^{3}+3 H_{1} \xi^{2}+2 H_{2} \xi+H_{3}}{-\xi\left(\xi^{4}+H_{1} \xi^{3}+H_{2} \xi^{2}+H_{3} \xi+H_{4}\right)}\right]\right|_{\tau_{2}=\tau_{0}}\right. \\
& \left.+\left.\operatorname{Re}\left[\frac{3 I_{1} \xi^{2}+2 I_{2} \xi+I_{3}}{\xi\left(I_{1} \xi^{3}+I_{2} \xi^{2}+I_{3} \xi+I_{4}\right)}\right]\right|_{\xi=i \omega_{0}}\right]=\operatorname{sign}\left[\frac{4 \omega_{0}^{6}+3 L_{1} \omega_{0}^{4}+2 L_{2} \omega_{0}^{2}+L_{3}}{\left(I_{2} \omega_{0}^{2}-I_{4}\right)^{2}+\left(I_{1} \omega_{0}^{3}-I_{3} \omega_{0}\right)^{2}}\right] \\
= & \operatorname{sign}\left[\frac{\Psi^{\prime}\left(\omega_{0}^{2}\right)}{\left(I_{2} \omega_{0}^{2}-I_{4}\right)^{2}+\left(I_{1} \omega_{0}^{3}-I_{3} \omega_{0}\right)^{2}}\right]=\operatorname{sign}\left[\frac{\left.\Psi_{0}\right)}{\left(I_{2} \omega_{0}^{2}-I_{4}\right)^{2}+\left(I_{1} \omega_{0}^{3}-I_{3} \omega_{0}\right)^{2}}\right]
\end{aligned}
$$

Thus, $\operatorname{sign}\left\{\mathrm{dRe} \xi\left(\tau_{2}\right) /\left.\mathrm{d} \tau_{2}\right|_{\tau_{2}=\tau_{0}}\right\}=\operatorname{sign}\left\{\Psi^{\prime}\left(z_{0}\right)\right\}$.

Summarizing the above and the Hopf bifurcation theorem [20] allows us to state the following results.

Theorem 6. If $R_{0}>1$ and (21) are satisfied, then the following results hold:

(i) If the conditions $(a)-(c)$ are all not satisfied, then the infection equilibrium $Q^{*}$ is locally asymptotically stable for all delay $\tau_{2} \geq 0$, which is called the delayindependent stability

(ii) If one of the conditions $(a)-(c)$ is satisfied, then the infection equilibrium $Q^{*}$ is locally asymptotically stable for all $\tau \in\left(0, \tau_{0}\right)$

(iii) If one of the conditions $(a)-(c)$ is satisfied and $\Psi^{\prime}\left(z_{0}\right) \neq 0$, then the transversality condition holds and model (1) undergoes a Hopf bifurcation at infection equilibrium $Q^{*}$ when $\tau_{2}=\tau_{0}$

From Lemma 2, we see that, to ensure the condition of transversality, it is mandatory that the positive roots of equation (36) are simple. First, we need the following lemma given by Hattaf [21].

Lemma 3. Let $P(x)$ be a polynomial of degree 4 with real coefficients:

(i) If the quartic equation $P(x)=0$ has only a single positive and simple root $x_{1}$, then $P^{\prime}\left(x_{1}\right)>0$

(ii) If the quartic equation $P(x)=0$ has only two positive and simple roots $x_{1}$ and $x_{2}$ (setting $x_{2}<x_{1}$ ), then $P^{\prime}\left(x_{1}\right)>0$ and $P^{\prime}\left(x_{2}\right)<0$

(iii) If the quartic equation $P(x)=0$ has only three positive and simple roots $x_{3}<x_{2}<x_{1}$, then $P^{\prime}\left(x_{1}\right)>0, P^{\prime}\left(x_{2}\right)<0$, and $P^{\prime}\left(x_{3}\right)>0$

(iv) If the quartic equation $P(x)=0$ has only four positive roots $x_{4}<x_{3}<x_{2}<x_{1}$, then $P^{\prime}\left(x_{1}\right)>0$, $P^{\prime}\left(x_{2}\right)<0, P^{\prime}\left(x_{3}\right)>0$, and $P^{\prime}\left(x_{4}\right)<0$
Theorem 7. Assume that $R_{0}>1$ and (21) holds. Define $\tau_{2, k_{0}}^{(n)}$ by (45).

(i) If equation (36) has only a single positive and simple root $z_{1}$, then $Q^{*}$ is locally asymptotically stable for $\tau_{2} \in\left(0, \tau_{2,1}^{(0)}\right)$ and unstable for $\tau_{2}>\tau_{2,1}^{(0)}$. Besides, $a$ Hopf bifurcation happens when $\tau_{2}>\tau_{2,1}^{(n)}, n \in \mathbb{N}$.

(ii) If equation (36) has only two positive and simple roots $z_{1}$ and $z_{2}$ with $z_{2}<z_{1}$, then there exists a finite number of intervals such that if the delay $\tau_{2}$ is fixed in these intervals, the equilibrium $Q^{*}$ is locally asymptotically stable, while unstable if $\tau_{2}$ does not belong to the ones. In this case, $Q^{*}$ changes from stability to instability.

(iii) If equation (36) has a minimum of three positive and simple roots, then there exists at least one stability change.

Proof. According to Theorem 5, $Q^{*}$ is locally asymptotically stable for $\tau_{2}=0$. Then, equation (31) has complex roots with negative real parts for $\tau_{2}=0$. If equation (36) has only one positive and simple root $z_{1}$, then $\pm i \omega_{1}$ is a pair of purely imaginary roots of equation (31) with $\tau_{2}=\tau_{2,1}^{(n)}$.

By applying Lemmas 2 and 3, we obtain

$$
\operatorname{sign}\left\{\left.\frac{\mathrm{dRe} \xi\left(\tau_{2}\right)}{\mathrm{d} \tau_{2}}\right|_{\tau_{2}=\tau_{2,1}^{(n)}}\right\}=\operatorname{sign}\left\{\Psi^{\prime}\left(z_{0}\right)\right\}>0 .
$$

Then, all roots of (31) have negative real parts for $\tau_{2} \in\left[0, \tau_{2,1}^{(0)}\right)$ and it has at least one root with positive real part for $\tau_{2}>\tau_{2,1}^{(0)}$. Therefore, we obtain $(i)$.

For (ii), we have $z_{2}<z_{1}$. From (44), we find that there exists $l \in \mathbb{N}$ such that $\tau_{2,1}^{(l)}-\tau_{2,1}^{(l-1)}=\left(2 \pi / \omega_{1}\right)<\left(2 \pi / \omega_{2}\right)=$ $\tau_{2,2}^{(l)}-\tau_{2,2}^{(l-2)}$. From Lemma 2 , we obtain that $\Psi^{\prime}\left(z_{1}\right)>0$ and $\Psi^{\prime}\left(z_{2}\right)<0$. Hence, $\operatorname{dRe} \xi\left(\tau_{2}\right) /\left.\mathrm{d} \tau_{2}\right|_{\tau_{2}=\tau_{2,1}^{(n)}}>0$ and $\operatorname{dRe} \xi\left(\tau_{2}\right) /$ $\left.\mathrm{d} \tau_{2}\right|_{\tau_{2}=\tau_{2,2}^{(n)}}<0$. We deduce that $l$ switches from stability to instability when the parameters $\tau_{2,1}^{(0)}<\tau_{2,2}^{(0)}<\tau_{2,1}^{(1)}<\cdots<\tau_{2,1}^{(l-1)}$ $<\tau_{2,2}^{(l-1)}<\tau_{2,1}^{(l)} ; \quad Q^{*}$ is locally asymptotically stable when 
$\tau_{2} \in\left[0, \tau_{2,1}^{(0)}\right] \cup\left[\tau_{2,2}^{(0)}, \tau_{2,1}^{(1)}\right] \cup, \ldots, \cup\left[\tau_{2,2}^{(l-1)}, \tau_{2,1}^{(l)}\right]$ and unstable when $\quad \tau_{2} \in\left[\tau_{2,1}^{(0)}, \tau_{2,2}^{(0)}\right] \quad \cup\left[\tau_{2,1}^{(1)}, \tau_{2,2}^{(1)}\right] \cup, \ldots, \cup\left[\tau_{2,1}^{(l-1)}, \tau_{2,2}^{(l-1)}\right] \cup$ $\left[\tau_{2,1}^{(l)},+\infty\right]$. This demonstrates (ii) and, additionally, we can undoubtedly obtain the outcome yielding (iii).

\section{Application}

The purpose of this section is to illustrate our theoretical results to the following model, which is a special case of system (1) by letting $f(U, I, C)=\left(\beta_{1} U / 1+\alpha_{1} C\right)$ and $g(U, I)=\left(\beta_{2} U / 1+\alpha_{2} I\right)$ :

$$
\left\{\begin{array}{l}
\dot{U}=s-d_{1} U(t)-\frac{\beta_{1} U(t) C(t)}{1+\alpha_{1} C(t)}-\frac{\beta_{2} U(t) I(t)}{1+\alpha_{2} I(t)} \\
\dot{I}=\frac{\beta_{1} U(t) C(t)}{1+\alpha_{1} C(t)}+\frac{\beta_{2} U(t) I(t)}{1+\alpha_{2} I(t)}-d_{2} I(t), \\
\dot{C}=v I\left(t-\tau_{1}\right)-d_{3} C(t)-p A(t) C(t), \\
\dot{A}=\sigma+q A\left(t-\tau_{2}\right) C\left(t-\tau_{2}\right)-d_{4} A(t),
\end{array}\right.
$$

where $\alpha_{1}$ and $\alpha_{2}$ are positive constants that measure the saturation effect, $\beta_{1}$ is the virus-to-cell infection rate, and $\beta_{2}$ is the cell-to-cell transmission rate. Apparently, the hypotheses $\left(\mathrm{H}_{0}\right)-\left(\mathrm{H}_{3}\right)$ hold and we have

$$
\begin{array}{r}
\left(1-\frac{f(U, I, C)}{f\left(U, I^{*}, C^{*}\right)}\right)\left(\frac{f\left(U, I^{*}, C^{*}\right)}{f(U, I, C)}-\frac{C}{C^{*}}\right)=\frac{-\alpha_{1}\left(C-C^{*}\right)^{2}}{C^{*}\left(1+\alpha_{1} C\right)\left(1+\alpha_{1} C^{*}\right)} \leq 0, \\
\left(1-\frac{f\left(U^{*}, I^{*}, C^{*}\right) g(U, I)}{f\left(U, I^{*}, C^{*}\right) g\left(U^{*}, I^{*}\right)}\right)\left(\frac{f\left(U, I^{*}, C^{*}\right) g\left(U^{*}, I^{*}\right)}{f\left(U^{*}, I^{*}, C^{*}\right) g(U, I)}-\frac{I}{I^{*}}\right)=\frac{-\alpha_{2}\left(I-I^{*}\right)^{2}}{I^{*}\left(1+\alpha_{2} I\right)\left(1+\alpha_{2} I^{*}\right)} \leq 0 .
\end{array}
$$

Therefore, hypothesis (21) is verified. From Theorems 4 and 5 , we have the following result.

\section{Corollary 1}

(i) If $R_{0} \leq 1$, then the infection-free equilibrium $Q_{f}$ of system (50) is globally asymptotically stable

(ii) If $R_{0}>1$, then the infection-free equilibrium $Q_{f}$ becomes unstable and the chronic infection equilibrium $Q^{*}$ of system (50) is globally asymptotically stable for $\tau_{2}=0$

Furthermore, theorem 7 holds true for system (50).

\section{Conclusion}

In this paper, we have presented a delayed CHIKV infection model with general incidence functions that include various forms existing in the literature. Initially, we have examined the nonnegativity, boundedness of the solutions, and the existence of equilibria. By building appropriate Lyapunov function, utilizing Lyapunov-LaSalle invariance principle and Hopf bifurcation theory, we have demonstrated the following outcomes: (i) when $R_{0} \leq 1$, the infection-free equilibrium $Q_{f}$ is globally asymptotically stable for any time delays $\tau_{1} \geq 0$ and $\tau_{2} \geq 0$, which naturally implies that the virus is cleared and the infection vanishes; (ii) when $R_{0}>1$ and $\left(H_{4}\right)$ holds, the chronic infection equilibrium $Q^{*}$ is globally asymptotically stable for anytime delay $\tau_{1} \geq 0$ and $\tau_{2}=0$, meaning that the infection perseveres in the host; (iii) when $\tau_{2}>0$ and $\tau_{1}=0$, we obtain the sufficient conditions on the existence of Hopf bifurcation at $Q^{*}$.

\section{Data Availability}

The data used to support the findings of this study are available from the corresponding author upon request.

\section{Conflicts of Interest}

The authors declare that they have no conflicts of interest.

\section{References}

[1] Y. Dumont and F. Chiroleu, "Vector control for the Chikungunya disease," Mathematical Biosciences and Engineering: $M B E$, vol. 7, no. 2, pp. 313-345, 2010.

[2] L. F. P. Ng, "Immunopathology of chikungunya virus Infection: lessons learned from patients and animal models," Annual Review of Virology, vol. 4, no. 1, pp. 413-427, 2017.

[3] C. Caglioti, E. Lalle, C. Castilletti, F. Carletti, M. R. Capobianchi, and L. Bordi, "Chikungunya virus infection: an overview," The New Microbiologica, vol. 36, no. 3, pp. 211-227, 2013.

[4] H. Delatte, G. Gimonneau, A. Triboire, and D. Fontenille, "Influence of temperature on immature development, survival, longevity, fecundity, and gonotrophic cycles ofAedes 
albopictus, vector of chikungunya and dengue in the Indian ocean," Journal of Medical Entomology, vol. 46, no. 1, pp. 33-41, 2009.

[5] P. Renault, J. L. Solet, D. Sissoko et al., "A major epidemic of chikungunya virus infection on Reunion Island, France, 20052006," The American Journal of Tropical Medicine and Hygiene, vol. 77, no. 4, pp. 727-731, 2007.

[6] D. Fischer, S. M. Thomas, J. E. Suk et al., "Climate change effects on chikungunya transmission in Europe: geospatial analysis of vector's climatic suitability and virus' temperature requirements," International Journal of Health Geographics, vol. 51, pp. 1-12, 2013.

[7] Ministry of Social Affairs and Health, Instruction $\mathrm{N}^{\circ}$ DGS/ VSS1/2017/128, http://circulaire.legifrance.gouv.fr/index. php?action $=$ afficherCirculaire \&hit $=1 \& \mathrm{r}=42120$.

[8] Y. Wang and X. Liu, "Stability and Hopf bifurcation of a within-host chikungunya virus infection model with two delays," Mathematics and Computers in Simulation, vol. 138, pp. 31-48, 2017.

[9] A. M. Elaiw, T. O. Alade, and S. M. Alsulami, "Analysis of within-host CHIKV dynamics models with general incidence rate," International Journal of Biomathematics, vol. 11, no. 5, pp. 1-25, 2018.

[10] M. Marsh and A. Helenius, "Virus entry: open sesame," Cell, vol. 124, no. 4, pp. 729-740, 2006.

[11] Q. Sattentau, "Avoiding the void: cell-to-cell spread of human viruses," Nature Reviews Microbiology, vol. 6, no. 11, pp. 815-826, 2008.

[12] W. Mothes, N. M. Sherer, J. Jin, and P. Zhong, "Virus cell-tocell transmission," Journal of Virology, vol. 84, no. 17, pp. 8360-8368, 2010.

[13] P. Zhong, L. M. Agosto, J. B. Munro, and W. Mothes, "Cell-tocell transmission of viruses," Current Opinion in Virology, vol. 3, no. 1, pp. 44-50, 2013.

[14] K. Hattaf and N. Yousf, "A generalized virus dynamics model with cell-to-cell transmission and cure rate," Advances in Difference Equations, vol. 2016, p. 174, 2016.

[15] K. Hattaf and N. Yousfi, "Qualitative analysis of a generalized virus dynamics model with both modes of transmission and distributed delays," International Journal of Differential Equations, vol. 2018, Article ID 9818372, 7 pages, 2018.

[16] A. M. Elaiw, S. E. Almalki, and A. Hobiny, "Global properties of saturated chikungunya virus dynamics models with cellular infection and delays," Advances in Differ Equations, vol. 476, pp. 1-33, 2019.

[17] H. Besbassi, Z. E. Rhoubari, K. Hattaf, and N. Yousfi, "Global dynamics of a generalized chikungunya virus," in Book: Trends in Biomathematics: Mathematical Modeling for Health, Harvesting, and Population Dynamics, pp. 107-117, Springer, Berlin, Germany, 2019.

[18] J. K. Hale and S. M. Verduyn Lunel, Introduction to Functional Differential Equations, Springer, New York, NY, USA, 1993.

[19] J. P. LaSalle, The Stability of Dynamical Systems, Regional Conference Series in Applied Mathematics, SIAM, Philadelphia, PA, USA, 1976.

[20] J. K. Hale, Theory of Functional Differential Equations, Springer, New York, NY, USA, 1977.

[21] K. Hattaf, "Global stability and Hopf bifurcation of a generalized viral infection model with multi-delays and humoral immunity," Physica A: Statistical Mechanics and Its Applications, vol. 545, 2020. 